\title{
Nash Social Welfare Approximation for Strategic Agents
}

\author{
SIMINA BRÂNZEI, Hebrew University of Jerusalem \\ VASILIS GKATZELIS, Drexel University \\ RUTA MEHTA, University of Illinois at Urbana-Champaign
}

The fair division of resources among strategic agents is an important age-old problem that has led to a rich body of literature. At the center of this literature lies the question of whether there exist mechanisms that can implement fair outcomes, despite the agents' strategic behavior. A fundamental objective function used for measuring the fairness of an allocation is the geometric mean of the agents' values, known as the Nash social welfare (NSW). This objective function is maximized by widely known solution concepts such as Nash bargaining and the competitive equilibrium with equal incomes.

In this work we focus on the question of (approximately) implementing this objective. The starting point of our analysis is the Fisher market, a fundamental model of an economy, whose benchmark is precisely the (weighted) Nash social welfare. We begin by studying two extreme classes of valuations functions, namely perfect substitutes and perfect complements, and find that for perfect substitutes, the Fisher market mechanism yields a constant approximation: at most 2 and at least $e^{\frac{1}{e}}(\approx 1.44)$. However, for perfect complements, the Fisher market mechanism does not work well, its bound degrading linearly with the number of players.

Strikingly, the Trading Post mechanism-an indirect market mechanism also known as the Shapley-Shubik game-has significantly better performance than the Fisher market on its own benchmark. Not only does Trading Post achieve an approximation of 2 for perfect substitutes, but this bound holds for any concave utilities, and it becomes essentially optimal for perfect complements, where it reaches $(1+\epsilon)$ for any $\epsilon>0$. Moreover, we show that all the Nash equilibria of the Trading Post mechanism are pure (hence the approximation factors extend to all Nash equilibria), and satisfy an important notion of individual fairness known as proportionality.

CCS Concepts: • Theory of computation $\rightarrow$ Quality of equilibria; Market equilibria;

Additional Key Words and Phrases: Nash social welfare; Fisher market; Trading post

\section{INTRODUCTION}

The question of allocating resources among multiple participants in a way that is fair is as old as human society itself [52], with some of the earliest recorded instances dating back to more than 2500 years ago ${ }^{1}$. The mathematical study of fair division began with the work of Steinhaus during the second world war, which led to an extensive and growing body of work on fair division

\footnotetext{
${ }^{1}$ See, e.g., Hesiod's Theogony, where a protocol known as Cut and Choose is mentioned.

Simina was supported by ISF grant 1435/14 administered by the Israeli Academy of Sciences and Israel-USA Bi-national Science Foundation (BSF) grant 2014389, and the I-CORE Program of the Planning and Budgeting Committee and The Israel Science Foundation. This project has received funding from the European Research Council (ERC) under the European Union's Horizon 2020 research and innovation programme (grant agreement No 740282). Vasilis was supported by NSF grants CCF-1408635, CCF-1216073, and CCF-1161813. This work was done in part while the authors were research fellows at the Simons Institute for the Theory of Computing.

Permission to make digital or hard copies of all or part of this work for personal or classroom use is granted without fee provided that copies are not made or distributed for profit or commercial advantage and that copies bear this notice and the full citation on the first page. Copyrights for components of this work owned by others than ACM must be honored. Abstracting with credit is permitted. To copy otherwise, or republish, to post on servers or to redistribute to lists, requires prior specific permission and/or a fee. Request permissions from permissions@acm.org.

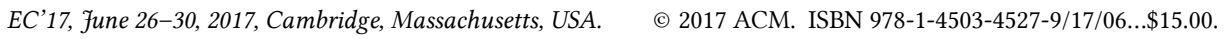

DOI: http://dx.doi.org/10.1145/3033274.3085143
} 
protocols within economics and political science, e.g., [6, 9, 52, 61, 65]. Recent years have seen an increased amount of work on fair division coming from computer science (see, e.g., [10, Part II]), partly motivated by problems related to allocating computational resources-such as CPU, memory, and bandwidth-among the users of a computing system. This work has focused on settings with both divisible goods (e.g., [11, 14, 20, 24, 57, 59]) and indivisible ones (e.g., [4, 16, 23, 60]).

One of the basic questions underlying the fair division problem is that of defining fairness to begin with, and a large body of work in economics, particularly social choice theory, is concerned with this very question, with numerous solution concepts proposed in response. Our fairness concept of choice herein is the Nash social welfare (NSW), which dates back to the fifties $[45,54]$ and has been proposed by Nash as a solution for bargaining problems, using an axiomatic approach. This objective aims to choose an outcome $\mathbf{x}$ maximizing the geometric mean of the utilities $\left(u_{i}(\mathbf{x})\right)$ of the $n$ participating agents and, like other standard welfare objectives, it is captured by a family of functions known as generalized (power) means:

$$
M_{p}(\mathbf{x})=\left(\frac{1}{n} \sum_{i}\left[u_{i}(\mathbf{x})\right]^{p}\right)^{1 / p} .
$$

In particular, the NSW corresponds to $M_{0}(\mathbf{x})$, the limit of $M_{p}(\mathbf{x})$ as $p$ goes to zero, i.e., $\left(\Pi_{i} u_{i}(\mathbf{x})\right)^{\frac{1}{n}}$.

While an extended treatment of the NSW can be found, for example, in [52], we highlight a fundamental property of the NSW objective, namely that it achieves a natural compromise between individual fairness and efficiency. Two other well-studied functions captured by $M_{p}(\mathbf{x})$ are the $(i)$ egalitarian ( $\max -\mathrm{min}$ ) objective attained as $p \rightarrow-\infty$, and (ii) utilitarian (average) objective attained at $p=1$, which correspond to extreme fairness and extreme efficiency, respectively. However, the former may cause vast inefficiencies, while the latter can completely neglect how unhappy some agents might be. The NSW objective lies between these two extremes and strikes a natural balance between them, since maximizing the geometric mean leads to more balanced valuations, but without neglecting efficiency.

The highly desired fairness and efficiency trade-off that the NSW objective provides can be verified via its close connection with market equilibrium outcomes in the Fisher market modelone of the fundamental resource allocation models in mathematical economics. This model was developed by Fisher [8] and studied in an extensive body of literature [17, 21, 28, 32, 33, 36-38, 44, 56]. The basic setting involves a seller who brings multiple divisible goods to the market and a set of buyers equipped with monetary endowments (budgets). The goal of the seller is to extract as much money as possible from the buyers, by charging money for the goods (through prices), while each buyer aims to acquire the best possible bundle of goods at the given prices. A market equilibrium is an outcome where supply meets demand, and has been shown to exist for very general models of an economy. When the buyers have the same budgets, this outcome is known as a competitive equilibrium from equal incomes (CEEI) [64]. For a broad family of valuations, including the ones considered in this paper, the market equilibrium allocation is known to maximize the NSW objective when the budgets are equal. In other words, the seller's goal can be achieved by computing the allocation of the goods that maximizes the NSW which, in turn, implies the desired price for each good.

A fundamental problem in implementing the Nash social welfare objective is informational: the seller needs to know the valuations of the participants. When these valuations are private information of the buyers, a natural candidate is the mechanism induced by the Fisher market, known as the Fisher market mechanism: ask the buyers to report their valuations, and then compute the NSW maximizing allocation based on the reports. Unfortunately, it is well known that buyers can feign different interests and eventually get better allocations $[1,5,12,18,19]$. This strategic 
behavior can result in the mechanism computing market equilibria with respect to preferences having little to do with reality, leading to unfair allocations. In this work we address the following basic question:

How well does the Fisher market mechanism optimize its own objective-the Nash social welfare-when the participants are strategic? Are there better mechanisms?

This question falls under the general umbrella of implementation theory [27], and particularly, of implementing markets [7, 31, 41, 53, 58]. In this literature, the goal is to identify mechanisms (game forms) for which the set of Nash equilibria coincides with the set of market equilibrium allocations for every possible state of the world [27]. In general this can be achieved only in the limit, as the number of players goes to infinity and every player is infinitesimal compared to the entire economy [30].

In this work we show that even for small markets there exist classic mechanisms achieving outcomes that closely approximate the optimal NSW on every instance while simultaneously guaranteeing individual fairness. We measure the quality of a mechanism using the Price of Anarchy (PoA) [55], defined as the ratio between the optimal NSW and the NSW of the worst Nash equilibrium outcome obtained by the mechanism.

\subsection{Our Results}

We study the question of approximately implementing the NSW objective starting with two extensively studied classes of valuations, namely linear (or additive) and Leontief [21, 36, 55], two extremes, and generalize many of our results to arbitrary concave valuations. Recall that additive valuations capture goods that are perfect substitutes, i.e., that can replace each other in consumption, such as Pepsi and Coca-Cola. Leontief valuations capture perfect complements, i.e., goods that have no value without each other, such as left and right shoes.

In addition to the aggregate measure of wealth captured through the Nash welfare, we will analyze mechanisms that guarantee proportionality, one of the fundamental fairness notions in fair division. Proportionality requires that every agent $i$ gets at least a fraction $B_{i} / \mathcal{B}$ of its utility for everything, where $B_{i}$ is its budget and $\mathcal{B}$ the sum of all budgets.

Our first set of results concerns the Fisher market mechanism, which collects the bidders valuations in the form of bids and then computes the market equilibrium based on those bids:

THeOREM (informal) Any Nash equilibrium of the Fisher market mechanism approximate the NSW objective within a factor of 2 for linear valuations. For Leontief valuations, the approximation degrades linearly with the number of players. Every Nash equilibrium of the mechanism is proportional for all concave utilities.

These bounds reveal significant differences between the quality of the Nash equilibria of the Fisher market mechanism for complements and substitutes.

Much more strikingly, we find that a classic mechanism known as Trading Post, originally introduced by Shapley and Shubik [1977] and studied in a long line of work in different scenarios [30, 43, 46, 49], offers very strong guarantees. At a high level, rather than collecting the players' preferences and computing the market equilibrium, the Trading Post mechanism gives each participant direct control over how to spend its budget. Once the agents choose how to distribute their budget over the available goods, they receive a fraction from each good that is proportional to the amount they spent on it. Our results for the Trading Post mechanism are: 
Theorem (informal) Any Nash equilibrium of the Trading Post mechanism approximates the NSW objective within a factor of 2 for all concave valuations. For Leontief valuations, the Trading Post mechanism achieves in the equilibrium an approximation of $1+\epsilon$ for every $\epsilon>0$. Moreover, all the Nash equilibria of Trading Post are pure and proportional for all concave utilities, while their existence is guaranteed for all CES utilities.

In other words, not only does the Trading Post mechanism achieve the same approximation as the Fisher market mechanism for additive valuations, but the bound holds for all concave valuations, and is arbitrarily close to the optimum for Leontief valuations! We view this as an important result that testifies to the usefulness and robustness of the Trading Post mechanism. A good Nash welfare approximation implies the geometric mean of values is high, and so utility is well distributed across the participants. Trading Post also ensures proportionality in any equilibrium outcome, thus the mechanism guarantees a surprising combination of both individual and aggregate fairness for very general utilities.

An interpretation of the Leontief result is that the Trading Post mechanism limits the extent to which an agent can affect the outcome, thus also limiting the extent to which things can go awry. Specifically, when an agent deviates in the Trading Post mechanism, this deviation has no effect on the way that the other agents are spending their money. On the other hand, an agent's deviation in the Fisher market mechanism can lead to a market equilibrium where the other agents' spending and allocation has changed significantly. In addition to this, in the Fisher market mechanism an agent can affect the price of an item even if the agent does not end up spending on that item in the final outcome. This is in contrast to the Trading Post mechanism where an agent can affect only the prices of the items that this agent is spending on, so the agents are forced to "put their money where their mouth is".

Finally, we prove that the set of mixed Nash equilibria of the Trading Post mechanism coincides with the set of pure Nash equilibria even with concave valuations, which extends our approximation bounds for this mechanism to mixed PoA. Moreover, both mechanisms achieve a classic notion of individual fairness in the equilibrium, namely proportionality. All of the above results work for the weighted version of the Nash social welfare, where agent $i$ has a $w_{i}$ fraction of the total budget, and the NSW objective becomes $\Pi_{i}\left[u_{i}(\mathbf{x})\right]^{w_{i}}$. In the process of obtaining a near optimal bound for Leontief in the Trading Post mechanism, we show that $\epsilon$-approximate market equilibria for Leontief utilities approximate its Nash social welfare by a factor of $\frac{1}{(1+\epsilon)}$. We believe that this as well as the technique that we developed to show the bounds of 2 may be of independent interest.

\subsection{Related Work}

The paper most closely related to our work is that of [24] which proposes truthful mechanisms for approximately maximizing the Nash social welfare objective. One of the truthful mechanisms that they propose, the Partial Allocation mechanism, guarantees a 2.718 approximation of the optimal NSW for both linear and Leontief valuations. In fact, the Partial Allocation mechanism guarantees that every agent receives a 2.718 approximation of the value that it would receive in the market equilibrium. But, in order to ensure truthfulness, this mechanism is forced to keep some of the goods unallocated, which makes it inapplicable for many real world settings. Complementing this mechanism, our work analyzes simple and well-studied mechanisms that allocate everything.

Most of the literature on fair division starting from the 1940's deals with the cake-cutting problem, which models the allocation of a divisible heterogeneous resource such as land, time, and mineral deposits, among agents with different preferences $[6,9,52,61,65]$. Some recent work has studied the agents' incentives in cake cutting. In particular, [20] study truthful cake-cutting with agents 
having piecewise uniform valuations and provide a polynomial-time mechanism that is truthful, proportional, and envy-free, while [51] shows that for general valuations there exists a protocol that is truthful in expectation, envy-free, and proportional for any number of players. The work of [48] shows that truthfulness comes at a significant cost in terms of efficiency for direct revelation mechanisms, while [14] show that the only strategyproof mechanisms in the standard query model for cake cutting are dictatorships (even for two players; a similar impossiblity holds for $n>2$ ). The standard cake cutting model assumes additive valuations, and so it does not capture resources with Leontief valuations, which we also analyze in this paper.

The resource allocation literature has seen a resurgence of work studying fair and efficient allocation for Leontief valuations [29, 40, 42, 57]. These valuations exhibit perfect complements and they are considered to be natural valuation abstractions for computing settings where jobs need resources in fixed ratios. [40] defined the notion of Dominant Resource Fairness (DRF), which is a generalization of the egalitarian social welfare to multiple types of resources. This solution has the advantage that it can be implemented truthfully for this specific class of valuations. [57] assessed DRF in terms of the resulting efficiency, showing that it performs poorly. [29] proposed an alternate fairness criterion called Bottleneck Based Fairness, which [42] subsequently showed is satisfied by the proportionally fair allocation. [42] also posed the study of incentives related to this latter notion as an interesting open problem. It is worth noting that [40] acknowledge that the CEEI, i.e., the NSW maximizing allocation would actually be the preferred fair division mechanism in their setting, and that the main drawback of this solution is the fact that it cannot be implemented truthfully. Our results show that the Trading Post mechanism can, in fact, approximate the CEEI outcome arbitrarily well, thus shedding new light on this setting.

The Trading Post mechanism, also known as the Shapley-Shubik game [62], has been studied in an extensive body of literature over the years, sometimes under very different names, such as Chinese auction [47], proportional sharing mechanism [35], and the Tullock contest in rent seeking $[34,50,63]$, the latter being a variant of the game with a different success probability for items that nobody bid on. Trading Post can also be interpreted as a congestion game (see, e.g., [39]), or an all-pay auction when the budgets are intrinsically valuable to the players.

The fact that, facing the Fisher mechanism, the agents may gain by bidding strategically is well known. [1] studied the agents' incentives and proved existence and structural properties of Nash equilibria for this mechanism. Extending this work, $[18,19]$ proved bounds on the extent to which an agent can gain by misreporting for various classes of valuation functions, including additive and Leontief. Finally, [12] showed bounds for the price of anarchy of this mechanism with respect to the social welfare objective, and [26] studied large markets under mild randomness and showed that this price of anarchy converges to one.

Finally, recent work on the NSW has revealed additional appealing properties of this objective. For indivisible items the NSW can be approximated in polynomial time [2, 3, 22, 23] and its optimal allocation is approximately envy-free [15]. On the other hand, for divisible items, it can be used as an intermediate step toward approximating the normalized social welfare objective [25].

\section{PRELIMINARIES}

Let $N=\{1, \ldots, n\}$ be a set of players (agents) and $M=\{1, \ldots, m\}$ a set of divisible goods. Player $i$ 's utility for a bundle of goods is represented by a non-decreasing non-negative concave valuation function $u_{i}:[0,1]^{m} \rightarrow \mathbb{R}_{+}$. An allocation $\mathrm{x}$ is a partition of the goods to the players such that $x_{i, j}$ represents the amount of good $j$ received by player $i$. Our goal will be to allocate all the resources fully; it is without loss of generality to assume that a single unit of each good is available, thus the set of feasible allocations is $\mathcal{F}=\left\{\mathbf{x} \mid x_{i, j} \geq 0\right.$ and $\left.\sum_{i=1}^{n} x_{i, j}=1\right\}$. 
Our measure for assessing the quality of an allocation is its Nash social welfare. At a given allocation $\mathbf{x}$ it is defined as follows

$$
\operatorname{NSW}(\mathbf{x})=\left(\prod_{i=1}^{n} u_{i}\left(\mathbf{x}_{i}\right)\right)^{\frac{1}{n}} .
$$

In order to also capture situations where the agents may have different importance or priority, such as clout in bargaining scenarios, we also consider the weighted version of the Nash social welfare objective. Note this is the objective maximized by the Fisher market equilibrium solution when the buyers have different budgets. We slightly abuse notation and refer to the weighted objective as the Nash social welfare (NSW) as well. If $B_{i} \geq 1$ is the budget of agent $i$ and $\mathcal{B}=\sum_{i=1}^{n} B_{i}$ is the total budget, the induced market equilibrium in Fisher's model maximizes the objective:

$$
\operatorname{NSW}(\mathbf{x})=\left(\prod_{i=1}^{n} u_{i}\left(\mathbf{x}_{i}\right)^{B_{i}}\right)^{\frac{1}{\mathcal{B}}} .
$$

Note that we get back the original definition when all players have the same budget. We would like to find mechanisms that maximize the NSW objective in the presence of strategic agents whose goal is to maximize their own utility.

We measure the quality of the mechanisms using the price of anarchy [55] with respect to the NSW objective. Given a problem instance $\mathcal{I}$ and some mechanism $\mathcal{M}$ that yields a set of pure Nash equilibria $E$, the price of anarchy (PoA) of $\mathcal{M}$ for $\mathcal{I}$ is the maximum ratio between the optimal NSWobtained at some allocation $\mathbf{x}^{*}$-and the NSW at an allocation $\mathbf{x} \in E: \operatorname{PoA}(\mathcal{M}, I)=\max _{\mathbf{x} \in E}\left\{\frac{\mathrm{NSW}\left(\mathbf{x}^{*}\right)}{\mathrm{NSW}(\mathbf{x})}\right\}$. The price of anarchy of $\mathcal{M}$ is the maximum of this value over all possible instances: $\max _{\mathcal{I}}\{\operatorname{PoA}(\mathcal{M}, \mathcal{I})\}$.

Valuation Functions. We start with two very common and extensively studied valuation functions that lie at the two extremes of a spectrum: perfect substitutes and perfect complements. For both, let $\boldsymbol{v}_{i}=\left(v_{i, 1}, \ldots, v_{i, m}\right) \in \mathbb{R}_{+}^{m}$ be a vector of valuations for agent $i$, where $v_{i, j}$ captures the liking of agent $i$ for good $j$.

Perfect substitutes, defined mathematically through linear (additive) valuations, represent goods that can replace each other in consumption, such as Pepsi and Coca-Cola. In the additive model, the utility of a player $i$ for bundle $\mathbf{x}_{i}$ is $u_{i}\left(\mathbf{x}_{i}\right)=\sum_{j=1}^{m} v_{i, j} \cdot x_{i, j}$.

Perfect complements, represented by Leontief utilities, capture scenarios where one good may have no value without the other, such as a left and a right shoe, or the CPU time and computer memory required for the completion of a computing task. In the Leontief model, the utility of a player $i$ for a bundle $\mathbf{x}_{i}$ is $u_{i}\left(\mathbf{x}_{i}\right)=\min _{j=1}^{m}\left\{x_{i, j} / v_{i, j}\right\}$; that is, player $i$ desires the items in the ratio $v_{i, 1}: v_{i, 2}: \ldots: v_{i, m}$. Coefficients can be rescaled freely in the Leontief model, so w.l.o.g. $v_{i, j} \geq 1$.

Finally, perfect substitutes and complements are extreme points of a much more general class of valuations known as CES (constant elasticity of substitution), mathematically defined as

$$
u_{i}\left(\mathbf{x}_{i}\right)=\left(\sum_{j=1}^{m} v_{i j} \cdot x_{i j}^{\rho}\right)^{\frac{1}{\rho}}
$$

where $\rho$ parameterizes the family, and $-\infty<\rho \leq 1, \rho \neq 0$. The Leontief and additive utilities are obtained when $\rho$ approaches $-\infty$ and equals 1 , respectively.

\section{THE FISHER MARKET MECHANISM}

In the Fisher market model, given prices $\boldsymbol{p}=\left(p_{1}, \ldots, p_{m}\right)$, each buyer $i$ demands a bundle $\boldsymbol{x}_{i}$ that maximizes her utility subject to her budget constraints; we call this an optimal bundle of buyer $i$ at 
prices $\boldsymbol{p}$. Prices $\boldsymbol{p}$ induce a market equilibrium if buyers get their optimal bundle and market clears. Formally, prices $\boldsymbol{p}$ and allocation $\boldsymbol{x}$ constitute a market equilibrium if

(1) Optimal bundle: $\forall i \in N$ and $\forall \boldsymbol{y}: \boldsymbol{y} \cdot \boldsymbol{p} \leq B_{i}, u_{i}\left(\mathbf{x}_{i}\right) \geq u_{i}(\boldsymbol{y})$

(2) Market clearing: Each good is fully sold or has price zero, i.e., $\forall j \in M, \sum_{j=1}^{m} x_{i, j} \leq 1$, and equality holds if $p_{j}>0$. Each buyer exhausts all its budget, i.e., $\forall i \in N, \sum_{j=1}^{m} x_{i, j} p_{j}=B_{i}$.

For linear and Leontief valuations, the market equilibria can be computed using the EisenbergGale (EG) convex program formulations that follow.

$$
\begin{array}{cl} 
& \multicolumn{1}{c}{\text { Linear }} \\
\max & \sum_{i=1}^{n} B_{i} \cdot \log u_{i} \\
\mathrm{s.t.} & u_{i}=\sum_{j=1}^{m} v_{i, j} x_{i, j}, \forall i \in N \\
& \sum_{i=1}^{n} x_{i, j} \leq 1, \quad \forall j \in M \\
& x_{i, j} \geq 0, \forall i \in N, j \in M
\end{array}
$$

\section{Leontief}

$$
\begin{aligned}
\max & \sum_{i=1}^{n} B_{i} \cdot \log u_{i} \\
\text { s.t. } & u_{i} \leq \frac{x_{i, j}}{v_{i, j}}, \forall i \in N, j \in M \\
& \sum_{i=1}^{n} x_{i, j} \leq 1, \quad \forall j \in M \\
& x_{i, j} \geq 0, \quad \forall i \in N, j \in M
\end{aligned}
$$

Let $p_{j}$ be the dual variable of the second inequality (for good $j$ ) in both cases, which corresponds to price of good $j$. Since due to strong duality Karush-Kuhn-Tucker (KKT) conditions capture solutions of the formulations, we get the following characterization for market equilibria.

For linear valuations, an outcome $(\boldsymbol{x}, \boldsymbol{p})$ is a market equilibrium if and only if,

$\operatorname{Lin}_{1} \forall i \in N$ and $\forall j \in M, x_{i, j}>0 \Rightarrow \frac{v_{i, j}}{p_{j}}=\max _{k \in M} \frac{v_{i, k}}{p_{k}}$.

$\operatorname{Lin}_{2} \forall i \in N, \sum_{j \in M} x_{i, j} p_{j}=B_{i} . \forall j \in M$, either $\sum_{i \in N} x_{i, j}=1$ or $p_{j}=0$.

For Leontief valuations, an outcome $(\boldsymbol{x}, \boldsymbol{p})$ is a market equilibrium if and only if.

$\mathbf{L e o}_{1} \forall i \in N$ and $\forall j \in M, v_{i, j}>0 \Rightarrow u_{i}=\frac{x_{i, j}}{v_{i, j}}=\frac{B_{i}}{\sum_{j \in M} v_{i, j} p_{j}}$. Note that $\sum_{j \in M} v_{i, j} p_{j}$ is the amount buyer $i$ has to spend to get unit utility.

Leo $_{2} \forall i \in N, \sum_{j \in M} x_{i, j} p_{j}=B_{i} . \forall j \in M$, either $\sum_{i \in N} x_{i, j}=1$ or $p_{j}=0$.

The Fisher market mechanism asks that the agents report their valuations and then it computes the market equilibrium allocation with respect to the reported valuations using the EG formulations.

Definition 3.1 (Fisher Market Mechanism). The Fisher Market Mechanism is such that:

- The strategy space of each agent $i$ consists of all possible valuations the agent may pose: $S_{i}=\left\{\mathbf{s}_{i} \mid \mathbf{s}_{i} \in \mathbb{R}_{\geq 0}^{m}\right\}$. We refer to an agent's strategy as a report.

- Given a strategy profile $\mathbf{s}=\left(\mathbf{s}_{i}\right)_{i=1}^{n}$, the outcome of the game is a market equilibrium of the Fisher market given by $\left\langle B_{i}, \mathbf{s}_{i}\right\rangle$, after removing the items $j$ for which $\sum_{i \in N} s_{i}(j)=0$. If there exists a market equilibrium $\mathcal{E}$ preferred by all the agents (with respect to their true valuations), then $\mathcal{E}$ is the outcome of the game on $\left\langle B_{i}, \mathbf{s}_{i}\right\rangle$. Otherwise, the outcome is any fixed market equilibrium.

It is well known that the buyers have incentives to hide their true valuations in a Fisher market mechanism. We illustrate this phenomenon through an example.

Example 3.2. Consider a Fisher market with players $N=\{1,2\}$, items $M=\{1,2\}$, additive valuations $v_{1,1}=1, v_{1,2}=0, v_{2,1}=v_{2,2}=0.5$, and budgets equal to 1 . If the players are truthful, the market equilibrium allocation is $\mathbf{x}_{1}=(1,0), \mathbf{x}_{2}=(0,1)$. However, if player 2 pretended that its value for item 2 is a very small $v_{2,2}^{\prime}=\epsilon>0$, then player 2 would not only get item 2 , but also a fraction of item 1.

In the rest of this section we study the performance of the Fisher mechanism when the goods are substitutes and complements, respectively. 


\subsection{Fisher Market: Perfect Substitutes}

In this section we study efficiency loss in the Fisher market due to strategic agents with additive valuations. We note that pure Nash equilibria in the induced game are known to always exist due to $[1] .^{2}$ Our first main result states that the Fisher market approximates the Nash Social Welfare within a small constant factor, even when the players are strategic.

Theorem 3.3. The Fisher Market Mechanism with linear valuations has price of anarchy at most 2.

We first prove a useful lemma, which bounds the change in prices due to a unilateral deviation in the Fisher market with additive valuations.

LemMa 3.4. Let $\mathbf{p}$ be the prices in a Fisher market equilibrium. Suppose that buyer i unilaterally changes its reported values to $\mathbf{v}_{i}^{\prime}$, leading to new market equilibrium prices $\mathbf{p}^{\prime}$. Then,

$$
\sum_{j: p_{j}^{\prime}>p_{j}} p_{j}^{\prime} \leq B_{i}+\sum_{j: p_{j}^{\prime}>p_{j}} p_{j} .
$$

Proof. Let $M^{+}, M^{-}$, and $M^{=}$be the sets of goods whose prices have strictly increased, strictly decreased, and remained unchanged, respectively, when transitioning from $\mathbf{p}$ to $\mathbf{p}^{\prime}$. If some player $k \neq i$ is buying any fraction of a good from $M^{-}$or $M^{-}$at $\mathbf{p}^{\prime}$, then the player cannot be buying anything from $M^{+}$at $\mathbf{p}^{\prime}$. To verify this fact, assume that player $k$ was spending on some item $\alpha \in M^{-} \cup M^{=}$at prices $\mathbf{p}$ and is now spending on some item $\beta \in M^{+}$at prices $\mathbf{p}^{\prime}$. Since both these allocations are market equilibria, $\mathbf{L i n}_{1}$ implies that $\frac{v_{k, \alpha}}{p_{\alpha}} \geq \frac{v_{k, \beta}}{p_{\beta}}$ at prices $\mathbf{p}$ and $\frac{v_{k, \alpha}}{p_{\alpha}^{\prime}} \leq \frac{v_{k, \beta}}{p_{\beta}^{\prime}}$ at prices $\mathbf{p}^{\prime}$. Since $p_{\alpha}^{\prime} \leq p_{\alpha}$ and $p_{\beta}^{\prime}>p_{\beta}$, this leads to the contradiction that $v_{k, \alpha}>v_{k, \alpha}$.

Thus, any player, other than $i$, who is buying goods from $M^{+}$at prices $\mathbf{p}^{\prime}$ had to be spending all its budget on these items at prices $\mathbf{p}$. This implies that the only reason why the sum of the prices of the goods in $M^{+}$could increase is because player $i$ is contributing more money on these items. Since the budget of $i$ is $B_{i}$, the total increase in these prices is at most $B_{i}$. This concludes the proof.

In addition to this, we will be using a folklore weighted arithmetic and geometric mean inequality stated in the following lemma.

Lemma 3.5. For any nonnegative numbers $\rho_{1}, \rho_{2}, \ldots, \rho_{n}$ and $w_{1}, w_{2}, \ldots, w_{n}$ with $W=\sum_{i=1}^{n} w_{i}$, we have $\left(\prod_{i=1}^{n} \rho_{i}^{w_{i}}\right)^{1 / W} \leq \frac{\sum_{i=1}^{n} \rho_{i} w_{i}}{W}$.

We can now prove the main theorem.

Proof. (of Theorem 3.3) Given a problem instance with additive valuations, let $\mathbf{x}^{*}$ be the allocation that maximizes the Nash social welfare-i.e., the market equilibrium allocation with respect to the true valuations-and $\tilde{\mathbf{x}}$ and $\tilde{\mathbf{p}}$ the allocation and prices respectively obtained under some Nash equilibrium of the market, where the players report fake valuations $\tilde{\mathbf{v}}$. Additionally, for each player $i$, let $\mathbf{x}^{i}$ be the allocation that would arise if every player $k \neq i$ reported $\tilde{\mathbf{v}}_{k}$ while $i$ reported its true value $v_{i j}$ for every item $j$ with $x_{i j}^{*}>0$, and zero elsewhere.

Since $\tilde{\mathbf{v}}$ is an equilibrium, this unilateral deviation of $i$ cannot increase its utility:

$$
u_{i}\left(\tilde{\mathbf{x}}_{i}\right) \geq u_{i}\left(\mathbf{x}_{i}^{i}\right)=\sum_{j=1}^{m} x_{i j}^{i} v_{i j} .
$$

\footnotetext{
${ }^{2}$ The existence is shown under a conflict-free tie breaking rule, which tries to allocate best bundle to as many agents as possible when there is a choice.
} 
Since $\mathbf{x}^{i}$ is a market equilibrium with respect to the reported values, according to the KKT condition $\left(\operatorname{Lin}_{1}\right), x_{i j}^{i}>0$ implies $v_{i j} / p_{j}^{i} \geq v_{i k} / p_{k}^{i}$ for any other item $k$, where $p_{j}^{i}$ is the price of item $j$ in $\mathbf{x}^{i}$. Also, the KKT condition $\left(\mathbf{L i n}_{2}\right)$ imply that $\sum_{j} x_{i j}^{i} p_{j}^{i}=B_{i}$ for every bidder $i$. Therefore, $\mathbf{x}_{i}^{i}$ provides $i$ at least as much value as any other bundle that $i$ can afford facing prices $p^{i}$, i.e., any bundle that costs at most $B_{i}$. In particular, consider the allocation $\mathrm{x}^{\prime}$ such that

For this allocation we have:

$$
x_{i j}^{\prime}=\frac{B_{i} x_{i j}^{*}}{\sum_{k=1}^{m} x_{i k}^{*} p_{k}^{i}} .
$$

$$
\sum_{j=1}^{m} x_{i j}^{\prime} p_{j}^{i}=\sum_{j=1}^{m}\left(\frac{B_{i} x_{i j}^{*}}{\sum_{k=1}^{m} x_{i k}^{*} p_{k}^{i}}\right) \cdot p_{j}^{i}=\frac{B_{i} \sum_{j=1}^{m} x_{i j}^{*} p_{j}^{i}}{\sum_{k=1}^{m} x_{i k}^{*} p_{k}^{i}}=B_{i} .
$$

Therefore, player $i$ can afford this bundle of items using the budget $B_{i}$, which implies that $\mathbf{x}_{i}^{i}$ provides $i$ at least as much value, i.e.,

$$
u_{i}\left(\mathbf{x}_{i}^{i}\right) \geq u_{i}\left(\mathbf{x}_{i}^{\prime}\right)=\sum_{j=1}^{m} v_{i, j} x_{i, j}^{\prime}=\frac{B_{i} u_{i}\left(\mathbf{x}_{i}^{*}\right)}{\sum_{j=1}^{m} x_{i, j}^{*} p_{j}^{i}}
$$

Let $\rho_{i}$ denote the ratio $\frac{u_{i}\left(\mathrm{x}^{*}\right)}{u_{i}\left(\tilde{\mathbf{x}}_{i}\right)}$. Using the Nash equilibrium inequality (2), we get:

$$
u_{i}\left(\tilde{\mathbf{x}}_{i}\right) \geq \frac{B_{i} u_{i}\left(\mathbf{x}_{i}^{*}\right)}{\sum_{j=1}^{m} x_{i, j}^{*} p_{j}^{i}} \Rightarrow \frac{u_{i}\left(\mathbf{x}^{*}\right)}{u_{i}\left(\tilde{\mathbf{x}}_{i}\right)} \leq \frac{\sum_{j=1}^{m} x_{i, j}^{*} p_{j}^{i}}{B_{i}} \Rightarrow \rho_{i} B_{i} \leq \sum_{j=1}^{m} x_{i, j}^{*} p_{j}^{i} .
$$

Using Lemma 3.4, we get:

$$
\rho_{i} B_{i} \leq \sum_{j=1}^{m} x_{i, j}^{*} p_{j}^{i} \leq \sum_{j: p_{j}^{i} \leq \tilde{p}_{j}} x_{i, j}^{*} \tilde{p}_{j}+\sum_{j: p_{j}^{i}>\tilde{p}_{j}} x_{i, j}^{*} p_{j}^{i} \leq B_{i}+\sum_{j=1}^{m} x_{i, j}^{*} \tilde{p}_{j},
$$

and summing over all players:

$$
\sum_{i=1}^{n} \rho_{i} B_{i} \leq \sum_{i=1}^{n}\left(B_{i}+\sum_{j=1}^{m} x_{i, j}^{*} \tilde{p}_{j}\right) \leq 2 \mathcal{B}
$$

Substituting $B_{i}$ for $w_{i}$ in Lemma 3.5 and using Inequality (3) yields:

$$
\left(\prod_{i=1}^{n} \rho_{i}^{B_{i}}\right)^{1 / \mathcal{B}} \leq \frac{\sum_{i=1}^{n} \rho_{i} B_{i}}{\mathcal{B}} \leq 2 .
$$

Next we show a lower bound for the price of anarchy of the Fisher mechanism. We construct a collection of problem instances whose PoA goes to $e^{1 / e}$ as number of players grows.

THEOREM 3.6. The Fisher Market mechanism with additive valuations has a price of anarchy no better than $e^{1 / e} \approx 1.445$.

Given some value of $n$, we construct a market with $n+2$ agents and $n+1$ goods. Fix an integer $k \leq n$; we will set its value later. Each player $i \leq k$ likes only good $i$, i.e., $v_{i, i}=1$ and all other $v_{i, j}=0$. On the other hand, every agent $i \in[k+1, n]$, apart from having $v_{i, i}=1$, also has some small, but positive, value $v_{i, j}=\epsilon$ for all items $j \leq k$. The rest of that agent's $v_{i, j}$ values are zero. Agent $n+1$ has a small but positive value $\epsilon^{\prime}$ for goods $j \in[k+1, n]$ and value 2 for good $n+1$. Finally, agent $n+2$ values only good $n+1$ at value 2 . Here $\epsilon<<\epsilon^{\prime}$, and we will set their values 
later. In the allocation where every agent $i \in[1, n]$ gets all of good $i$, while agents $n+1$ and $n+2$ share good $n+1$ equally, the NSW is equal to 1 . Next, we construct a Nash equilibrium strategy profile $s$ of the above market where the NSW approaches $(1 / e)^{1 / e}$ as $n \rightarrow \infty$.

We define a strategy profile $s$ where the first $k$ agents and the last agent, i.e., agent $n+2$, bid truthfully, while every bidder $i \in[k+1, n]$ misreports in a way such that it ends up spending some small amount $\delta=2 \epsilon^{\prime}$ on item $i$ and the rest of its budget, namely $(1-\delta)$, is equally divided on items in $[1, k]$. We later set a value for $\delta$ such that agent $n+1$ would want to buy only good $n+1$.

We now show that the above profile is a Nash equilibrium for carefully chosen values of $\epsilon$, and $\epsilon^{\prime}$. Note that, since bidders $i \in[1, k]$ and bidder $n+2$ bids truthfully and values just one item, each one of these bidders will spend all of its budget on the corresponding items, no matter what the remaining bidders report. Therefore, the price of items $j \in[1, k]$ and item $n+1$ will be exactly 1 if we exclude the spending of bidders $i \in[k+1, n+1]$, no matter what these bidders report. Also, if the price of items $j \in[k+1, n]$ is equal to $\delta$ in profile $s$, then this price will not drop below $\delta$, irrespective of what bidder $n+1$ reports. In the next two lemmas, we show that a deviation from $s$ does not help the bidders, even when the prices are held constant for the goods where an agent starts spending more money.

Lemma 3.7. If $\delta=2 \epsilon^{\prime}$ then, even if the prices of goods $j \in[k+1, n]$ are fixed at $\delta$, the $(n+1)^{t h}$ bidder has no incentive to spend money on any good other than good $n+1$.

Proof. If agent $n+1$ spends all of its budget on item $n+1$, then its price becomes 2 and the agent receives half of that item, i.e., a utility of 1 . On the other hand, if the agent spends at total of $\gamma>0$ on goods $j \in[k+1, n]$ then, even if the price of these items remains $\delta$, her utility would be $\epsilon^{\prime} \frac{\gamma}{\delta}+2 \frac{1-\gamma}{2-\gamma}$. For this to be strictly greater than 1 we have to have $\delta<2 \epsilon^{\prime}-\gamma \epsilon^{\prime}$, which contradicts our assumption that $\delta=2 \epsilon^{\prime}$.

Furthermore, using a similar analysis as that of Lemma 3.7, it follows that if agent $i \in[k+1, n]$ deviates in a way that decreases its spending on good $i$ to $2 \epsilon^{\prime}-\tau$ for $\tau>0$, then the Fisher market mechanism outcome will have the $(n+1)^{t h}$ agent spending at least $\frac{\tau}{1+\epsilon^{\prime}}$ on that item. Thus, such a deviation would cause agent $i$ to lose its monopoly on good $i$. Next, we show that this is not advantageous for agent $i$ if we set $\epsilon=\frac{1}{n^{4}}$ and $\epsilon^{\prime}=\frac{1}{n}$, which implies $\delta=\frac{2}{n}$.

LemMA 3.8. For any $\tau \geq 0$, if agent $i$ is spending $\delta-\tau$ on good $i$ and agent $(n+1)$ is spending $\frac{\tau}{1+\epsilon^{\prime}}$, when others are bidding according to $s$, then agent $i$ 's utility is maximized at $\tau=0$.

Proof. Note that at $\tau=0$, agent $i \in[k+1, n]$ is spending $\delta=2 \epsilon^{\prime}$ on good $i$ and, according to Lemma 3.7, agent $n+1$ would not be interested in spending on good $i$. Thus, agent $i$ is buying good $i$ exclusively and spends $\frac{1-\delta}{k}$ on each of the first $k$ goods. Thus the price each one of the first $k$ goods is $1+(n-k) \frac{1-\delta}{k}$. Agent $i$ 's utility at this allocation is

$$
1+\frac{\frac{1-\delta}{k}}{1+(n-k) \frac{1-\delta}{k}} k \epsilon=1+\frac{(1-\delta) k \epsilon}{k+(n-k)(1-\delta)}
$$

As mentioned above, if agent $i$ reduces spending on good $i$ by $\tau$, then the $(n+1)^{t h}$ agent will end up spending at least $\frac{\tau}{1+\epsilon^{\prime}}$ on this item. This may lead to increased prices for the first $k$ goods, but we show that this deviation would not benefit $i$ even if these prices remained the same. The utility of agent $i$ after such a deviation would be

$$
\frac{\delta-\tau}{\delta-\tau+\frac{\tau}{1+\epsilon^{\prime}}}+\frac{(1-\delta+\tau) k \epsilon}{k+(n-k)(1-\delta)}
$$


The latter utility is greater than the former only when

$$
\frac{\tau k \epsilon}{k+(n-k)(1-\delta)}>\frac{\frac{\tau}{1+\epsilon^{\prime}}}{\delta-\tau+\frac{\tau}{1+\epsilon^{\prime}}} \Rightarrow \epsilon>\frac{k+(n-k)(1-\delta)}{k\left(\delta+\delta \epsilon^{\prime}-\tau \epsilon^{\prime}\right)}>1,
$$

which contradicts the fact that $\epsilon=\frac{1}{n^{4}}$.

Lemmas 3.7 and 3.8 imply that $s$ is a Nash equilibrium. The price of the first $k$ goods at this Nash equilibrium is $1+\frac{(n-k)(1-\delta)}{k}=\frac{k+(n-k)(1-\delta)}{k}$. Thus, the utility of buyer $i \in[1, k]$, who spends all of its $\$ 1$ on $\operatorname{good} i$, is $u_{i}=\frac{k}{k+(n-k)(1-\delta)}$. On the other hand, the utility of buyer $i \in[k+1, n]$, who gets all of good $i$ and some of the first $k$ goods, is $u_{i}=1+\frac{(1-\delta) k \epsilon}{k+(n-k)(1-\delta)}$. Agents $(n+1)$ and $(n+2)$ get half of $\operatorname{good} n+1$ and thereby get utility of 1 each. Since $\epsilon=\frac{1}{n^{4}}$ and $\epsilon^{\prime}=\frac{1}{n}$, then $\forall i \in[1, k], \lim _{n \rightarrow \infty} u_{i}=\frac{k}{n}$ and $\forall i \in[k+1, n] \lim _{n \rightarrow \infty} u_{i}=1$. Thus, NSW at this bid profile as $n \rightarrow \infty$ is $\left(\frac{k}{n}\right)^{\frac{k}{n}}$, and thereby PoA is at least $\left(\frac{n}{k}\right)^{k / n}$. Letting $k=\frac{n}{e}$, this becomes $e^{1 / e}$, which concludes the proof of Theorem 3.6.

\subsection{Fisher Market: Perfect Complements}

On the other hand, the Fisher market with perfect complements has a price of anarchy that grows linearly with the number of players.

THeorem 3.9. The Fisher Market Mechanism with Leontief valuations has a price of anarchy of n, the number of players, and the bound is tight.

REMARK. We observe that PoA of Fisher Market Mechanism degrades continuously within CES as we move from additive to Leontief. For the complements range, i.e., $\rho<0$, the example achieving $n$ lower bound for Leontief can be modified to show that as $\rho$ decreases the PoA increases. The modification is in the Nash equilibrium profile, where agent $i$ reports $v_{i, i}=1+\epsilon$ instead of 1 for an appropriately chosen $\epsilon \geq 0$ depending on $\rho$ and number of players $n$.

\section{THE TRADING POST MECHANISM}

The important difference between the Trading Post mechanism and the Fisher Market mechanism is the strategy space of the agents. More precisely, unlike the Fisher market mechanism, where the agents' are asked to report their valuations, the Trading Post mechanism instead asks the agents to directly choose how to distribute their budgets. Once the agents have chosen how much of their budget to spend on each of the goods, the total spending on each good $j$ is treated as its price, and each agent $i$ is allocated a fraction of good $j$ proportional to the amount that $i$ is spending on $j$. Therefore, the strategy set of each player $i$ is $\mathcal{S}_{i}=\left\{\mathbf{b}_{i} \in\left[0, B_{i}\right]^{m} \mid \sum_{j=1}^{m} b_{i, j}=B_{i}\right\}$.

Given a bid profile $\mathbf{b}=\left(\mathbf{b}_{1}, \ldots, \mathbf{b}_{n}\right)$, the induced allocation is:

$$
x_{i, j}= \begin{cases}\frac{b_{i, j}}{\sum_{k=1}^{n} b_{k, j}} & \text { if } b_{i, j}>0 \\ 0 & \text { otherwise }\end{cases}
$$

In this section we analyze Trading Post for additive and Leontief valuations, extending a number of results to CES and arbitrary concave functions under a mild technical condition known as perfect competition, which states that for each good $j$, there exist at least two buyers that demand strictly positive amount of $j$ when the good is priced at zero. For additive and Leontief valuations, the condition is equivalent to $v_{i, j}>0, v_{k, j}>0$, for some $i, k \in N, i \neq k$. If this were not satisfied, we could either discard the good or give it away for free.

Our main results in this section are that the pure Nash equilibria of Trading Post approximate the NSW objective within a factor of 2 for linear valuations, and within a factor of $1+\epsilon$ for every $\epsilon>0$ for Leontief valuations. In fact we extend the approximation factor of 2 to CES and more 
generally to concave utilities. Moreover, in all of these cases, all the Nash equilibria of the game are pure and their existence is guaranteed for CES utilities.

\subsection{Trading Post: Perfect Substitutes}

The existence of pure Nash equilibria in the Trading Post mechanism for additive valuations was established by Feldman, Lai, and Zhang [35] under perfect competition. Without competition, even very simple games may not have pure Nash equilibria. To see this, consider for instance a game with two players, two items, and additive valuations $v_{1,1}=1, v_{1,2}=0, v_{2,1}=v_{2,2}=0.5$. Through a case analysis it can be seen that both players will compete for item 1, while player 2 is the only one that wants item 2, reason for which this player will successively reduce its bid for 2 to get a higher fraction from item 1 . However, in the limit of its bid for the second item going to zero, player 2 loses the item.

Our first result shows that Trading Post matches the Fisher market for additive valuations.

Theorem 4.1. The Trading Post Mechanism with linear valuations has price of anarchy at most 2.

Proof. Given a problem instance with linear valuations, let $\mathbf{x}^{*}$ be the allocation that maximizes the NSW and $p^{*}$ be the corresponding market equilibrium prices. Also, let $\tilde{\mathbf{x}}$ be the allocation in a Nash equilibrium where each player $i$ bids $\tilde{b}_{i}$ and the price of each item $j$ is $\tilde{p}_{j}=\sum_{i} \tilde{b}_{i, j}$.

For some player $i$, let $\mathbf{x}^{\prime}$ be the allocation that arises if every player $k \neq i$ bids $\tilde{\boldsymbol{b}}_{k}$ while agent $i$ unilaterally deviates to $b_{i, j}^{\prime}$ for each item $j$. In this deviation, player $i$ first withdraws all of its money, leaving the price of item $j$ to be $p_{j}^{\prime}=\tilde{p}_{j}-\tilde{b}_{i, j}$, and then it redistributes it by spending $b_{i, j}^{\prime}$ on each item $j$. The perfect competition condition ensures that, once player $i$ withdraws its money, the prices remain positive $\left(p_{j}^{\prime}>0, \forall j\right)$, as at least two agents are interested in each good. Let the new bid $b_{i, j}^{\prime}$ be such that for some $\beta_{i}>0$ and every item $j$ :

$$
\frac{b_{i, j}^{\prime}}{p_{j}^{\prime}+b_{i, j}^{\prime}}=\frac{x_{i, j}^{*}}{\beta_{i}}
$$

Bid $\mathbf{b}_{i}^{\prime}$ is implied by the solution of the following program.

$$
\min : \beta_{i} \quad \text { s.t.: } \quad \frac{1}{\beta_{i}}=\frac{b_{i, j}^{\prime}}{x_{i, j}^{*}\left(p_{j}^{\prime}+b_{i, j}^{\prime}\right)} \quad \text { and } \quad b_{i, j}^{\prime} \geq 0 \quad \forall j \in M ; \quad \sum_{j \in M} b_{i, j}^{\prime} \leq B_{i}
$$

Setting $\boldsymbol{b}_{i, j}^{\prime}=0$ and $\beta_{i}=\infty$ gives a feasible point in the above program, and therefore it has a minimum. The allocation induced by this unilateral deviation of $i$ is $x_{i, j}^{\prime}=\frac{b_{i, j}^{\prime}}{p_{j}^{\prime}+b_{i, j}^{\prime}}=\frac{x_{i, j}^{*}}{\beta_{i}}$. Therefore, the utility of player $i$ after this deviation is $u_{i}\left(\mathbf{x}^{*}\right) / \beta_{i}$. But the outcome $\tilde{\mathbf{x}}$ is a Nash equilibrium, so this deviation cannot yield a higher utility for $i$, which implies that $u_{i}(\tilde{\mathbf{x}}) \geq u_{i}\left(\mathbf{x}^{*}\right) / \beta_{i}$. By definition of $b^{\prime}$, we get $\sum_{j} b_{i, j}^{\prime}=\sum_{j} x_{i, j}^{\prime}\left(p_{j}^{\prime}+b_{i, j}^{\prime}\right)=B_{i}$; all the money is spent at optimum $\beta_{i}$. Therefore, replacing for $x_{i, j}^{\prime}=x_{i, j}^{*} / \beta_{i}$, we get

$$
B_{i} \beta_{i}=\sum_{j=1}^{m} x_{i, j}^{*}\left(p_{j}^{\prime}+b_{i, j}^{\prime}\right)
$$

Since $0 \leq x_{i, j}^{*} \leq 1$ and $p_{j}^{\prime} \leq \tilde{p}_{j}$ for each item $j$, for agent $i$ we have,

$$
\sum_{j=1}^{m} x_{i, j}^{*}\left(p_{j}^{\prime}+b_{i, j}^{\prime}\right) \leq \sum_{j=1}^{m} x_{i, j}^{*} b_{i, j}^{\prime}+\sum_{j=1}^{m} x_{i, j}^{*} \tilde{p}_{j} \leq B_{i}+\sum_{j=1}^{m} x_{i, j}^{*} \tilde{p}_{j} .
$$


Using Inequalities (5) and (6), and summing over all players gives

$$
\sum_{i=1}^{n} B_{i} \frac{u_{i}\left(\mathbf{x}_{i}^{*}\right)}{u_{i}\left(\tilde{\mathbf{x}}_{i}\right)} \leq \sum_{i=1}^{n} B_{i} \beta_{i} \leq \sum_{i=1}^{n}\left(B_{i}+\sum_{j=1}^{m} x_{i, j}^{*} \tilde{p}_{j}\right) \leq 2 \mathcal{B} .
$$

Using the inequality of weighted arithmetic and geometric means provided by Lemma 3.5 on $\beta_{i}$ 's of (7), we conclude that $\prod_{i=1}^{n}\left(\frac{u_{i}\left(\mathbf{x}_{i}^{*}\right)}{u_{i}\left(\tilde{\mathbf{x}}_{i}\right)}\right)^{B_{i} / \mathcal{B}}=\prod_{i=1}^{n}\left(\beta_{i}\right)^{B_{i} / \mathcal{B}} \leq 2$.

The next theorem complements the upper bound on the price of anarchy with a lower bound of approximately 1.445 .

Theorem 4.2. The Trading Post mechanism has a price of anarchy no better than $e^{1 / e} \approx 1.445$.

Proof. In order to prove this theorem we can use the same family of problem instances used in the proof of Theorem 3.6. In fact, verifying that the market equilibrium outcome induced by the strategy profile $s$ in that construction is a Nash equilibrium for the Trading Post mechanism as well is much more straightforward since an agent's deviation may affect only the way that particular agent ends up spending its budget.

The upper and lower bounds of Trading Post with additive valuations given by Theorems 4.1 and 4.2 are same as that of Fisher market mechanism. However, next we show this symmetry breaks as soon as we move away from additive valuations, and Trading Post fares significantly better.

\subsection{Trading Post: Perfect Complements}

We first characterize the precise conditions under which the Trading Post mechanism has exact pure Nash equilibria for Leontief utilities; the missing proofs of this section may be found in the full version [13].

Theorem 4.3. The Trading Post mechanism with Leontief utilities has pure Nash equilibria if and only if the corresponding market equilibrium prices are all strictly positive. When this happens, the Nash equilibrium utilities in Trading Post are unique and the price of anarchy is 1.

This theorem shows a correspondence between the pure Nash equilibria of Trading Post and the corresponding market equilibria with respect to the agents' (true) valuations. We observe however that existence of pure Nash equilibria in Trading Post is not guaranteed for Leontief utilities.

Example 4.4. Consider a game with two players and two items, where player 1 has values $v_{1,1}=v_{1,2}=0.5$ and player 2 has $v_{2,1}=0.9, v_{2,2}=0.1$. Assume there is a pure Nash equilibrium profile $\mathbf{b}$. Since both players require a non-zero amount from every item for their utility to be positive, we have that $b_{i, j}>0$ for all $i, j \in\{1,2\}$. Denote $b_{1}=b_{1,1}$ and $b_{2}=b_{2,1}$; then $b_{1,2}=1-b_{1}$ and $b_{2,1}=1-b_{2}$. Note that each player must receive the two items in the same ratio relative to its valuation; that is:

$$
u_{i}(\mathbf{b})=\left(\frac{b_{i}}{b_{1}+b_{2}}\right) \frac{1}{v_{i, 1}}=\left(\frac{1-b_{i}}{b_{1}+b_{2}}\right) \frac{1}{v_{i, 2}}
$$

Otherwise, if the two ratios were not equal, then a player could transfer weight among the items to improve the smaller fraction. Then the requirement in 8 are equivalent to the following equations:

$$
\left(\frac{b_{1}}{b_{1}+b_{2}}\right) \frac{1}{0.5}=\left(\frac{1-b_{1}}{b_{1}+b_{2}}\right) \frac{1}{0.5} \Longleftrightarrow b_{1}=b_{2}
$$

and

$$
\left(\frac{b_{2}}{b_{1}+b_{2}}\right) \frac{1}{0.9}=\left(\frac{1-b_{2}}{b_{1}+b_{2}}\right) \frac{1}{0.1} \Longleftrightarrow 8 b_{2}^{2}+8 b_{1} b_{2}=9 b_{1}+7 b_{2}
$$


Combining equations 9 and 10 , we get that $b_{1}=1$ and $b_{2}=1$, which contradicts the requirement that $b_{1}, b_{2} \in(0,1)$. Thus the equilibrium profile $\mathbf{b}$ cannot exist.

The issue illustrated by this example is that the Trading Post cannot implement market outcomes when there exist items priced at zero in the corresponding market equilibrium. This motivates us to introduce an entrance fee in the Trading Post mechanism, denoted by a parameter $\Delta>0$, which is the minimum amount that an agent needs to spend on an item in order to receive any of it. We denote the corresponding mechanism by $\mathcal{T P}(\Delta)$. The value of $\Delta$ can be arbitrarily small, so its impact on the outcome of the game is insignificant.

Formally, given a bid profile $\mathbf{b}=\left(\mathbf{b}_{1}, \ldots, \mathbf{b}_{n}\right)$, let $\overline{\mathbf{b}}=\left(\overline{\mathbf{b}}_{1}, \ldots, \overline{\mathbf{b}}_{n}\right)$ be the "effective" bid profile which, for every $i \in N$ and $j \in M$, satisfies $\bar{b}_{i, j}=b_{i, j}$ if $b_{i, j} \geq \Delta$, and $\bar{b}_{i, j}=0$ otherwise. Then, the bid profile $b$ yields the allocation:

$$
x_{i, j}=\frac{\bar{b}_{i, j}}{\sum_{k=1}^{n} \bar{b}_{k, j}} \text { if } \bar{b}_{i, j}>0, \quad \text { and } \quad x_{i, j}=0 \text { otherwise }
$$

Clearly, in any Nash equilibrium, we have that for every player $i$ and item $j$ we have that $b_{i, j} \geq \Delta$ or $b_{i, j}=0$ (the latter identity holds for those goods $j$ that are outside of player $i$ 's demand).

The main result of this section is that, for every $\epsilon \in(0,1 / m)$, the Trading Post mechanism with $\Delta \leq \epsilon / \mathrm{m}^{2}$ has a price of anarchy of at most $1+\epsilon$. We first show that Trading Post has a pure Nash equilibrium for Leontief utilities for every strictly positive entrance fee. The proof uses an application of Glicksberg's theorem for continuous games.

Theorem 4.5. The parameterized Trading Post mechanism $\mathcal{T P}(\Delta)$ is guaranteed to have a pure Nash equilibrium for every $\Delta>0$.

We start by defining a notion of approximate market equilibrium that will be useful

Definition 4.6 ( $\epsilon$-market equilibrium). Given a problem instance and some $\epsilon>0$, an outcome $(\mathbf{p}, \mathbf{x})$ is an $\epsilon$-market equilibrium if and only if: (i) All the goods with a positive price are completely sold. (ii) All the buyers exhaust their budget. (iii) Each buyer gets an $\epsilon$-optimal bundle at prices $\mathbf{p}$; that is, for every bundle $\mathbf{y} \in[0,1]^{m}$ that $i$ could afford at these prices $\left(\mathbf{p} \cdot \mathbf{y} \leq B_{i}\right)$, we have $u_{i}(\mathbf{y}) \leq u_{i}\left(\mathbf{x}_{i}\right)(1+\epsilon)$.

The following theorem states that for every small enough $\epsilon>0$, all the PNE of the Trading Post game with a small enough entrance fee correspond to $\epsilon$-market equilibrium outcomes.

Theorem 4.7. Let $\epsilon>0$. Then for every $0<\Delta<\min \left\{\frac{\epsilon}{m^{2}}, \frac{1}{m}\right\}$, every pure Nash equilibrium of the mechanism $\mathcal{T P}(\Delta)$ with Leontief valuations corresponds to an $\epsilon$-market equilibrium.

Proof. Let $\tilde{b}$ be a pure Nash equilibrium of $\mathcal{T P}(\Delta)$ and $\mathbf{x}$ the induced allocation. For each player $i$, let $D_{i}=\left\{j \in M \mid v_{i, j}>0\right\}$ be the set of items that $i$ requires, and let $m_{i}=\left|D_{i}\right|$. We also override notation and refer to $u_{i}(\mathbf{b})$ as the utility of player $i$ when the strategy profiles are $\mathbf{b}$.

First note that $\tilde{\boldsymbol{b}}_{i, j}>0$ for each player $i$ and item $j \in D_{i}$. If this were not the case, then player $i$ would get zero utility at strategy profile $\tilde{\boldsymbol{b}}$; this is worse than playing the uniform strategy $\mathbf{z}_{i}=\left(B_{i} / m, \ldots, B_{i} / m\right)$, which guarantees $i$ a positive value regardless of the strategies of the other players $\tilde{\boldsymbol{b}}_{-i}$, namely:

$$
u_{i}\left(\mathbf{z}_{i}, \tilde{b}_{-i}\right)=\min _{j \in D_{i}}\left\{\frac{z_{i, j}}{z_{i, j}+\sum_{k \neq i} \tilde{b}_{k, j}} \cdot \frac{1}{v_{i, j}}\right\} \geq \min _{j \in D_{i}}\left\{\frac{B_{i} / m}{B_{i} / m+\sum_{k \neq i} B_{k}} \cdot \frac{1}{v_{i, j}}\right\}>0
$$


For each player $i$ and item $j \in D_{i}$, denote the fraction of utility that $i$ derives from $j$ by:

$$
\phi_{i, j}=\frac{\tilde{b}_{i, j}}{\sum_{k=1}^{n} \tilde{b}_{k, j}} \cdot \frac{1}{v_{i, j}} .
$$

Then $u_{i}(\tilde{\boldsymbol{b}})=\min _{j \in D_{i}} \phi_{i, j}$. Sort the items in $D_{i}$ increasingly by their contribution to $i$ 's utility: $\phi_{i, i_{1}} \leq \phi_{i, i_{2}} \leq \ldots \leq \phi_{i, i_{m_{i}}}$; it follows that $u_{i}(\tilde{\boldsymbol{b}})=\phi_{i, i_{1}}$. Let $S_{i}=\left\{j \in D_{i} \mid \phi_{i, j}=\phi_{i, i_{1}}\right\}$ be the items received in the smallest fraction (equal to $i$ 's utility). If $S_{i}=M$, then the analysis is similar to the exact equilibrium case, where the prices are strictly positive. The difficult case is when $S_{i} \neq M$. Then player $i$ is getting a higher than necessary fraction from some resource $j \in M \backslash S_{i}$. Thus $i$ would improve by shifting some of the mass from item $j$ to the items in $S$. Since $\tilde{\boldsymbol{b}}$ is an equilibrium, no such deviation is possible. Then it must be the case that $\tilde{b}_{i, j}=\Delta$ for all $j \in D_{i} \backslash S_{i}$.

Now interpret the bids and allocation as a market equilibrium with Leontief utilities $\mathbf{v}$ and budgets $B_{i}$, by setting the prices to $\mathbf{p}=\left(p_{1}, \ldots, p_{m}\right)$, where $p_{j}=\sum_{i=1}^{n} \tilde{b}_{i, j}$ for all $j \in M$, and the allocation to $\mathbf{x}$, the same as the one induced by the bids $\tilde{b}$ in the trading post game. We argue that $(\mathbf{p}, \mathbf{x})$ is an $\epsilon$-market equilibrium. Clearly at the outcome $(\mathbf{p}, \mathbf{x})$ all the goods are sold and each buyer exhausts their budget. Moreover observe that all the prices are strictly positive. We must additionally show that each player gets an $\epsilon$-optimal bundle at $(\mathbf{p}, \mathbf{x})$.

Fix an arbitrary buyer $i$. Let $\mathbf{y}_{i}$ be an optimal bundle for $i$ given prices $\mathbf{p}$, and let $q_{i, j}$ be the amount of money spent by $i$ to purchase $y_{i, j}$ units of good $j$ at these prices. An upper bound on the optimal value $u_{i}\left(\mathrm{y}_{i}\right)$ is attained when buyer $i$ shifts all the money spent on purchasing items outside $S_{i}$ to purchase instead higher fractions from the items in $S_{i}$. Since the strategy profile b is an exact equilibrium in the game $\mathcal{T P}(\Delta)$, the amount of money spent by player $i$ on items outside $S_{i}$ is at most $(m-1) \Delta$; thus $i$ spends at most $B_{i}-(m-1) \Delta$ on the remaining items in $S_{i}$.

By an averaging argument, there exists a good $j \in S_{i}$ on which $i$ spends the greatest amount of its money, i.e. $\tilde{b}_{i, j} \geq \frac{B_{i}-(m-1) \Delta}{\left|S_{i}\right|}$. This will be the item for which the gain brought by the deviation in spending is modest. Formally, the maximum fraction of utility that $i$ can get from item $j-$ without decreasing the ratios at which the other items in $S_{i}$ are received-is:

$$
\begin{aligned}
\phi_{i, j}^{\prime} & =\frac{q_{i, j}}{p_{j} \cdot v_{i, j}} \leq \frac{\tilde{b}_{i, j}+(m-1) \Delta}{\left(\sum_{k=1}^{n} \tilde{b}_{k, j}\right) \cdot v_{i, j}}=\phi_{i, j}+\frac{(m-1) \Delta}{\left(\sum_{k=1}^{n} \tilde{b}_{k, j}\right) \cdot v_{i, j}} \\
& \leq \phi_{i, j}+\frac{\tilde{b}_{i, j} \cdot \epsilon}{\left(\sum_{k=1}^{n} \tilde{b}_{k, j}\right) \cdot v_{i, j}}=\phi_{i, j} \cdot(1+\epsilon)=u_{i}\left(\mathbf{x}_{i}\right)(1+\epsilon)
\end{aligned}
$$

where in the inequalities we additionally used that $\Delta<\epsilon^{2} / m, B_{i} \geq 1 \forall i \in N$, and $S_{i} \leq m-1$. The identities hold since item $j$ is in the tight set $S_{i}$. Then $u_{i}\left(\mathbf{y}_{i}\right) \leq \phi_{i, j}^{\prime} \leq u_{i}\left(\mathbf{x}_{i}\right)(1+\epsilon)$. Thus each player gets an $\epsilon$-optimal bundle, and so (p, $\mathbf{x})$ is an $\epsilon$-market equilibrium.

The following theorem, which we believe is of independent interest, states that in Fisher markets with Leontief utilities, approximate market equilibria are close to exact equilibria in terms of their Nash Social Welfare.

THEOREM 4.8. The Nash social welfare at an $\epsilon$-market equilibrium for Leontief utilities is at least a $\frac{1}{(1+\epsilon)}$ factor of the optimal Nash social welfare.

Finally, we can state the main result of this section. 
Theorem 4.9. For every $\epsilon>0$, the Trading Post game $\mathcal{T P}(\Delta)$ with entrance fee $0<\Delta<$ $\min \left\{\frac{\epsilon^{2}}{m}, \frac{1}{m}\right\}$ has a price of anarchy of $1+\epsilon$, even for arbitrary budgets.

Proof. By Theorem 4.7, every PNE of $\mathcal{T P}(\Delta)$ corresponds to an $\epsilon$-market equilibrium. By Theorem 4.8, every $\epsilon$-market equilibrium attains at least a fraction $\frac{1}{1+\epsilon}$ of the optimal Nash social welfare. Thus, the price of anarchy of $\mathcal{T P}(\Delta)$ is $1+\epsilon$, which completes the proof.

\subsection{Trading Post: Beyond Perfect Substitutes and Complements}

In general market equilibrium theory the valuations of the agents are considered to be arbitrary concave, non-decreasing and non-negative functions. Next we extend the upper bound of 2 achieved by Trading Post to this most general setting. The missing proofs are in the full version [13].

Theorem 4.10. The Trading Post game with concave valuations has price of anarchy at most 2.

Moreover, the existence of pure Nash equilibria holds for all CES utilities.

Theorem 4.11. The Trading Post game with no minimum bid has exact pure Nash equilibria for all CES utilities with perfect competition and $\rho \in(-\infty, 1]$.

\subsection{Trading Post: Beyond Pure Nash Equilibria}

A natural remaining question is whether there exist mixed Nash equilibria with bad Nash social welfare. In the case of Trading Post mechanism we rule out this case by showing that all its Nash equilibria are pure, be it for linear, Leontief, or concave valuations. Details of this result may be found in the full version [13], where we show the following theorem.

Theorem 4.12. For markets with linear utilities, every Nash equilibrium of the corresponding Trading Post game is pure. Further, the result extends to concave valuations if a mild condition of enough competition is satisfied. For markets with Leontief utilities, every Nash equilibrium of the corresponding $\Delta$ Trading Post game TP $(\Delta)$, for $\Delta>0$, is pure.

Given such an efficiency achieved by Trading Post mechanism one may wonder if these mechanisms in-fact have unique equilibrium. We rule this out through constructions for additive and Leontief valuations, given in the full version [13].

\section{FAIRNESS GUARANTEES}

Finally, for concave utilities all the Nash equilibria of the two mechanisms satisfy an important notion of individual fairness: proportionality. That is, each player $i$ achieves a fraction of at least $B_{i} / \mathcal{B}$ of its maximum utility in any PNE. For equal budgets, the guarantee is $1 / n$.

THeOREM 5.1. Every Nash equilibrium of the Fisher market mechanism with concave utilities, and of the Trading Post mechanism with concave and strictly increasing utilities is proportional. Moreover, for every $0<\Delta<1 / m$, every Nash equilibrium of the parameterized Trading Post game TP $(\Delta)$ with concave and strictly increasing utilities is approximately proportional, guaranteeing at least $\frac{B_{i}}{\mathcal{B}}\left(1-\rho_{i}\right)$ of the optimum for each player $i$, where $\rho_{i}=\frac{\Delta \cdot(m-1)}{B_{i}}, B_{i}$ is the player's budget, $\mathcal{B}$ the sum of budgets, and $m$ the number of items.

\section{DISCUSSION}

Our results show great differences between the Fisher market mechanism and Trading Post, with Trading Post simultaneously achieving a Nash social welfare within a factor of two of the optimum and fairness (in the form of proportionality) for all concave utilities. Existence of pure Nash equilibria in Trading Post is guaranteed for all CES utilities. 
Our most unexpected and important contribution is for Leontief utilities, where Trading Post approximates the NSW arbitrarily close, while still guaranteeing fair outcomes to each individual. This result may have implications beyond theory, since Leontief utilities have played a starring role in the emerging literature on allocating computer resources. One of the most influential papers in this literature [40] suggested that the NSW maximizing outcome would be the ideal solution, its only drawback being the fact that it cannot be reached in the presence of strategic participants.

An interesting open question is that of achieving good approximations of the NSW objective using truthful, but non-wasteful, mechanisms.

\section{REFERENCES}

[1] B. Adsul, Ch. Sobhan Babu, J. Garg, R. Mehta, and M. Sohoni. 2010. Nash equilibria in Fisher market. In SAGT. 30-41.

[2] N. Anari, T. Mai, S. Oveis Gharan, and V. V. Vazirani. 2016. Nash Social Welfare for Indivisible Items under Separable, Piecewise-Linear Concave Utilities. ArXiv e-prints (Dec. 2016). arXiv:cs.GT/1612.05191

[3] N. Anari, S. Oveis-Gharan, A. Saberi, and M. Singh. 2017. Nash social welfare, matrix permanent, and stable polynomials. In ITCS.

[4] H. Aziz and S. Mackenzie. 2016. A Discrete and Bounded Envy-free Cake Cutting Protocol for Four Agents. In STOC.

[5] M. Babaioff, B. Lucier, N. Nisan, and R. Paes Leme. 2014. On the Efficiency of the Walrasian Mechanism. In EC. 783-800.

[6] J.B. Barbanel. 2004. The Geometry of Efficient Fair Division. Cambridge Univ. Press.

[7] C. Bevia, L. Corchón, and S. Wilkie. 2003. Implementation of the Walrasian correspondence by market games. Review of Economic Design 7 (2003), 429-442.

[8] W. C. Brainard and H. E. Scarf. 2000. How to compute equilibrium prices in 1891. Cowles Foundation Discussion Paper $1270(2000)$.

[9] S. Brams and A. Taylor. 1996. Fair Division: from cake cutting to dispute resolution. Cambridge University Press, Cambridge.

[10] F. Brandt, V. Conitzer, U. Endriss, J. Lang, and A. D. Procaccia. 2016. Handbook of Computational Social Choice. Cambridge University Press.

[11] S. Brânzei, I. Caragiannis, D. Kurokawa, and A.D. Procaccia. 2016. An Algorithmic Framework for Strategic Fair Division. In $A A A I$.

[12] S. Brânzei, Y. Chen, X. Deng, A. Filos-Ratsikas, S. Frederiksen, and J. Zhang. 2014. The Fisher Market Game: Equilibrium and Welfare. In $A A A I$. 587-593.

[13] S. Branzei, V. Gkatzelis, and R. Mehta. 2017. Nash Social Welfare Approximation for Strategic Agents. (2017). arxiv/abs/1607.01569.

[14] S. Brânzei and P. B. Miltersen. 2015. A Dictatorship Theorem for Cake Cutting. In IfCAI. 482-488.

[15] I. Caragiannis, D. Kurokawa, H. C. Moulin, A. D. Procaccia, N. Shah, and J. Wang. 2016. The Unreasonable Fairness of Maximum Nash Welfare. In EC. 305-322.

[16] D. Chakrabarty, J. Chuzhoy, and S. Khanna. 2009. On Allocating Goods to Maximize Fairness. In FOCS. 107-116.

[17] N. Chen, Xiatie Deng, Xiaoming Sun, and Andrew Yao. 2004. Fisher Equilibrium Price with a class of Concave Utility Functions. In ESA. 169-179.

[18] N. Chen, X. Deng, H. Zhang, and J. Zhang. 2012. Incentive Ratios of Fisher Markets. In ICALP.

[19] N. Chen, X. Deng, and J. Zhang. 2011. How Profitable Are Strategic Behaviors in a Market?. In ESA.

[20] Y. Chen, J.K. Lai, D.C. Parkes, and A.D. Procaccia. 2013. Truth, justice, and cake cutting. Games and Economic Behavior 77, 1 (2013), 284-297.

[21] B. Codenotti and K. Vardarajan. 2004. Equilbrium for Markets with with Leontief Utilities. In ICALP.

[22] R. Cole, N.R. Devanur, V. Gkatzelis, K. Jain, T. Mai, V.V. Vazirani, and S. Yazdanbod. 2017. Convex Program Duality, Fisher Markets, and Nash Social Welfare. In EC.

[23] R. Cole and V. Gkatzelis. 2015. Approximating the Nash Social Welfare with Indivisible Items. In STOC. 371-380.

[24] R. Cole, V. Gkatzelis, and G. Goel. 2013. Mechanism design for fair division: allocating divisible items without payments. In EC. 251-268.

[25] R. Cole, V. Gkatzelis, and G. Goel. 2013. Positive results for mechanism design without money. In AAMAS. $1165-1166$.

[26] R. Cole and Y. Tao. 2016. Large Market Games with Near Optimal Efficiency. In Proceedings of the 2016 ACM Conference on Economics and Computation, EC 2016,. 791-808.

[27] P. Dasgupta, P. Hammond, and E. Maskin. 1979. The implementation of social choice rules: Some general results on incentive compatibility. The Review of Economic Studies 46, 2 (1979), 185-216.

[28] N. Devanur, C.H. Papadimitriou, A. Saberi, and V. V. Vazirani. 2008. Market equilibrium via a primal-dual algorithm for a convex program. $7 A C M 55,5$ (2008). 
[29] D. Dolev, D.G. Feitelson, J.Y. Halpern, R. Kupferman, and N. Linial. 2012. No justified complaints: on fair sharing of multiple resources. In ITCS. 68-75.

[30] P. Dubey and J. Geanakoplos. 2003. From Nash to Walras via Shapley-Shubik. fournal of Mathematical Economics 39,5 (2003), 391-400.

[31] P. Dubey and M. Shubik. 1978. The noncooperative equilibria of a closed trading economy with market supply and bidding strategies. JET 17 (1978), 1-20.

[32] B.C. Eaves. 1976. A finite algorithm for the linear exchange model. F. Math. Econ. 3 (1976), 197-203.

[33] E. Eisenberg and D. Gale. 1959. Consensus of subjective probabilities: the Pari-Mutuel method. The Annals of Mathematical Statistics 30 (1959), 165-168.

[34] H. Fang. 2002. Lottery versus all-pay auction models of lobbying. Public Choice 112, 3-4 (2002), 351-71.

[35] M. Feldman, K. Lai, and L. Zhang. 2009. The Proportional-Share Allocation Market for Computational Resources. ITPDS 20, 8 (2009).

[36] D. Gale. 1960. Theory of Linear Economic Models. McGraw Hill, N.Y.

[37] D. Gale. 1976. The Linear exchange Model. J. Math. Econ. 3 (1976), 205-209.

[38] R. Garg, S. Kapoor, and V. V. Vazirani. 2004. An Auction-Based Market Equilbrium Algorithm for the Separable Gross Substitutibility Case. In APPROX.

[39] C. Georgiou, T. Pavlides, and A. Philippou. 2006. Network uncertainty in selfish routing. In International Parallel and Distributed Processing Symposium.

[40] A. Ghodsi, M. Zaharia, B. Hindman, A. Konwinski, S. Shenker, and I. Stoica. 2011. Dominant resource fairness: fair allocation of multiple resource types. In NSDI.

[41] G. Giraud. 2003. Strategic market games: an introduction. Journal of Mathematical Economics 39 (2003), 355-375.

[42] A. Gutman and N. Nisan. 2012. Fair allocation without trade. In AAMAS. 719-728.

[43] M. O. Jackson and J. Peck. 1999. Asymmetric information in a competitive market game: Reexamining the implications of rational expectations. Econ. Theory 13 (1999), 603-628.

[44] K. Jain, V. V. Vazirani, and Y. Ye. 2005. Market Equilibrium for Homothetic, Quasi-Concave Utilities and Economies of Scale in Production. In SODA.

[45] M. Kaneko and K. Nakamura. 1979. The Nash Social Welfare Function. Econometrica 47, 2 (1979), 423-435.

[46] C. G. Korpeoglu and S. E. Spear. 2015. The Market Game with Production: Coordination Equilibrium and Price Stickiness. (2015). CMU, Tepper School of Business.

[47] A. Matros. 2007. Chinese auctions. (2007). Mimeo, University of Pittsburgh.

[48] A. Maya and N. Nisan. 2012. Incentive Compatible Two Player Cake Cutting. In WINE.

[49] J.-F. Mertens and S. Sorin (eds). 2013. Game-theoretic methods in general equilibrium analysis. Springer Science \& Business Media.

[50] B. Moldovanu and A. Sela. 2001. The optimal allocation of prizes in contests. AER 91, 3 (2001), 542-558.

[51] E. Mossel and O. Tamuz. 2010. Truthful Fair Division. In SAGT. 288-299.

[52] H. Moulin. 2003. Fair Division and Collective Welfare. The MIT Press.

[53] S. Nakamura. 1990. A feasible Nash implementation of Walrasian equilibria in the two-agent economy. Economics Letters 34, 1 (1990), 5-9.

[54] J. Nash. 1950. The Bargaining Problem. Econometrica 18, 2 (April 1950), 155-162.

[55] N. Nisan, T. Roughgarden, E. Tardos, and V. Vazirani. 2007. Algorithmic Game Theory. Cambridge University Press.

[56] J. B. Orlin. 2010. Improved algorithms for computing Fisher's market clearing prices. In STOC. 291-300.

[57] D.C. Parkes, A.D. Procaccia, and N. Shah. 2012. Beyond dominant resource fairness: extensions, limitations, and indivisibilities. In EC. 808-825.

[58] A. Postlewaite and D. Schmeidler. 1986. Implementation in Differential Information Economies. $7 E T 39$ (1986), 14-33.

[59] A.D. Procaccia. 2013. Cake Cutting: Not Just Child's Play. Commun. ACM 56, 7 (2013), $78-87$.

[60] A.D. Procaccia and Junxing Wang. 2014. Fair enough: guaranteeing approximate maximin shares. In EC. 675-692.

[61] J.M. Robertson and W.A. Webb. 1998. Cake-cutting algorithms - be fair if you can. A K Peters. I-X, 1-181 pages.

[62] L. Shapley and M. Shubik. 1977. Trade using one commodity as a means of payment. fournal of Political Economy 85(5) (1977), 937-968.

[63] G. Tullock. 1980. Efficient rent-seeking. In Toward a theory of the rent-seeking society, G. Tullock J.M. Buchanan, R.D. Tollison (Ed.). Texas A. \& M, University Press.

[64] H.R. Varian. 1974. Equity, envy, and efficiency. Journal of Economic Theory 9, 1 (1974), 63-91.

[65] H.P. Young. 1995. Equity. Princeton University Press. 